\title{
Foreword: computational special focus issue
}

\author{
Chérif F Matta*,1 \& Michael C Hutter ${ }^{1}$ \\ ${ }^{1}$ Center for Bioinformatics, Building E2.1, Saarland University, D-66123 Saarbruecken, Germany \\ *Author for correspondence: michael.hutter@bioinformatik.uni-saarland.de
}

First draft submitted: 4 May 2018; Accepted for publication: 4 May 2018; Published online: 20 July 2018

Keywords: artificial intelligence • deep learning • drug discovery • machine learning • PAINS • pharmacophore models • quantitative structure-activity relationship

When did computational approaches appear first in the history of medicinal chemistry? There must have been approaches even before large mainframe computers were built in the 1960s. Was it the connection between lipophilicity of organic substances to their toxic and narcotic action independently described by $\mathrm{HH}$ Meyer and $\mathrm{E}$ Overton, which eventually led to the concept of quantitative structure-activity relationship (QSAR) [1,2]? Or was it P Ehrlich's dogma that substances have to bind in order to exert their function ('corpora non agunt nisi fixata') which can be viewed as an ancestor to the pharmacophore models [3]?

We can go back even further, to AC Brown and T Frazer, who tried to link the structure and function of alkaloids [4]. Even today, we are doing our best to find the right descriptors, which can connect chemical structure to biological function. Only the procedures(rather than the abstract concepts) have much changed, regarding both experimental set-up and computational methods. Machine learning methods, such as neural networks, random forests and support vectors machines have meanwhile become well-established in the toolbox of researchers, with more advanced developments, such as deep learning and artificial intelligence, already making their way into preclinical development.

This special issue of Future Medicinal Chemistry intends to highlight some of the recent developments and emerging fields in computational medicinal chemistry. Tackling 'big data' is more than a buzzword these days. How can we derive useful information from the vast junk of ever-growing unstructured data? Pattern recognition and perception are the key terms for any algorithm dealing with this task, no matter if it is machine learning or the more complex artificial intelligence. Deep learning refers to the extension of common artificial neural networks by adding either more hidden layers or intensifying the interconnections between neurons of the layers, now allowing signals to be sent back to neurons in previous layers. Both approaches are thought to model the functionality of organic neural networks more closely, hence can increase performance.

The editorial by J Mitchell gives some realistic assessments of what is already achievable and most likely to be seen with learning methods in the field of drug discovery [5]. Examples of the application of deep learning for the prediction of activity and toxicology can be found in J Bajorath's contribution [6]. Nevertheless, even an 'old' method, such as QSAR modeling is far from being outdated. In contrast to big data, with QSAR modeling, we often deal with small datasets comprising less than 100 compounds. Using careful application and validation, Stålring et al. demonstrate the effectiveness of this approach for human liver microsomal activity [7].

Limits on the application of prediction methods, either from the site of the experiment or the algorithmic background are outlined in the review of M Hutter [8]. Again, it is all about data, but in this case how better prediction regarding toxicity, sites of metabolism, binding to antitargets and docking results are held back by a lack of information. An algorithmic prediction can only be as good as the data it is trained on, thus errors in data give rise to uncertainties. There is need to have a reliable dataset at hand.

Curating high-quality compilations of data often requires tedious manual work, digging into original literature and being able to judge potential sources of errors which may have been overlooked by the authors themselves. $\mathrm{R}$ Wang and co-workers contributed a new benchmark set to evaluate scoring functions for docking, comprising 273 protein-protein complexes [9]. Since modulation of protein-protein interaction is an emerging field in drug discovery, this is a particularly valuable addition for further theoretical development.

For experimentalists, it is beneficial to know which compounds are likely to give wrong results, such as the so-called pan-assay interference compounds. These substances typically appear as frequent hitters for a multitude 
of targets. Approaches to dissect those 'bad actors', which are actually false positives (such as due to the formation of aggregates) from true promiscuous compounds are discussed by Stork and Kirchmair [10].

Not only novel computational techniques are of interest, but also emerging or overlooked targets. Histone deacetylases have gained attention in the last two decades, due to their regulatory function in the transcription of genetic information from DNA. This also affects epigenetic repression and control of the cell cycle, which renders them promising targets in related diseases (e.g., chronic myeloid leukemia). Jha and co-workers used a Laplacian-corrected Bayesian model to extract frequently found substructures from fingerprints, while discriminating inhibitors from noninhibitors [11]. Selectivity is also a major problem, due to the presence of 11 isoforms of human histone deacetylases giving rise to several cavities in the vicinity of the main pocket. The group of W Sippl managed to consider these aspects in their pharmacophore model, which is able to describe most known inhibitors [12].

The EGFR, which is an exploited target for the same cancer type, also has a kinase domain. Here, the emergence of drug-resistant mutations requires new strategies. Rastelli and co-workers report a rapid docking protocol to identify allosteric inhibitors, leading to the experimental validation of 92 compounds, of which one possesses a scaffold differing from existing allosteric EGFR inhibitors [13].

In comparison to cancer, obesity looks more like a manageable condition, which is also the subject of intense research. The presence of almost 300 inhibitors of pancreatic lipase requires different QSAR approaches. As with increasing data sizes, it becomes difficult to find descriptors that show sufficiently high correlation. This is typically due to the structural dissimilarity of the substances causing the data to cluster into distinct subgroups, with inevitable discrepancies in experimental data between different laboratories. Kumar and Chaupan have devised a method to obtain hybrid descriptors, applying Monte Carlo optimization to cope with active and rare structural attributes [14]. Novel compounds proposed by this approach were predicted to exhibit higher activities than their parent structures.

There are instances where trend detection techniques fail dramatically, such as activity cliffs whereby a small change in structure can lead to a drastic change in activity. In these cases, highly accurate quantum mechanicalbinding energies can be crucial to understanding the binding of an inhibitor to its host macromolecular receptor. Traditional crystallography typically delivers the (all important) three-dimensional structure of an inhibitor-host complex but no information on the binding strength. Such energetic information can now be made accessible from 'Quantum Crystallography', the potential applications of which in medicinal chemistry are highlighted by CF Matta and L Massa [15].

\section{Financial \& competing interests disclosure}

The author has no relevant affiliations or financial involvement with any organization or entity with a financial interest in or financial conflict with the subject matter or materials discussed in the manuscript. This includes employment, consultancies, honoraria, stock ownership or options, expert testimony, grants or patents received, pending, or royalties.

No writing assistance was utilized in production of the manuscript.

\section{References}

1. Meyer H. Zur Theorie der Alkoholnarkose. Erste Mittheilung. Welche Eigenschaft der Anasthetica bedingt ihre narkotische Wirkung? Arch. Exp. Pathol. Pharmacol. 42, 109-118 (1899).

2. Overton CE. Studien über die Narkose, Fischer, Jena, Germany (1901).

3. Bosch F, Rosich L. The contributions of Paul Ehrlich to pharmacology: a tribute on the occasion of the centenary of his nobel prize. Pharmacology 82, 171-179 (2008).

4. Crum-Brown A, Fraser TR. On the connection between chemical constitution and physiological action. Part. I. on the physiological action of the salts of the ammonium bases, derived from strychnia, brucia, thebaia, codeia, morphia and nicotia. Trans. R. Soc. Edinburgh 25, 151-203 (1868).

5. Mitchell J. AI in pharmaceutical research and development. Future Med. Chem. 10(13), 1529-1531 (2018).

6. Bajorath J. Data analytics and deep learning in medicinal chemistry. Future Med. Chem. 10(13), 1541-1543 (2018).

7. Stålring J, Sohlenius-Sternbeck AK, Terelius Y, Parkes K. Confident application of a global human liver microsomal activity QSAR. Future Med. Chem. 10(13), 1575-1588 (2018).

8. Hutter M. The current limits in virtual screening and property prediction. Future Med. Chem. 10(13), 1623-1635 (2018).

9. Han L, Yang Q, Li Y, Wang R. Development of a new benchmark for assessing the scoring functions applicable to protein-protein interaction. Future Med. Chem. 10(13), 1555-1574 (2018). 
10. Stork C, Kirchmair J. PAIN(S) relievers for medicinal chemists: how computational methods can assist in hit evaluation. Future Med. Chem. 10(13), 1533-1535 (2018).

11. Amin SA, Adhikari N, Jha T. Diverse classes of HDAC8 inhibitors: in search of molecular fingerprints that regulate activity. Future Med. Chem. 10(13), 1589-1602 (2018).

12. Melesina J, Praetorius L, Simoben CV, Robaa D, Sippl W. Design of selective HDAC inhibitors: rethinking classical pharmacophore. Future Med. Chem. 10(13), 1537-1540 (2018).

13. Caporuscio F, Tinivella A, Restelli V et al. Identification of small-molecule EGFR allosteric inhibitors by high-throughput docking. Future Med. Chem. 10(13), 1545-1553 (2018).

14. Kumar A, Chaupan S. Use of SMILES and molecular graph based descriptors in prediction and design of pancreatic lipase inhibitors. Future Med. Chem. 10(13), 1603-1622 (2018).

15. Matta C, Massa L. Quantum crystallography in medicinal chemistry. Future Med. Chem. 10(13), 1525-1527 (2018). 
